\title{
INTEGRAL PARAMETERS AND THE TOTAL MASS \\ OF OPEN CLUSTERS
}

(Abstract)

A. E. PISKUNOFF

Astronomical Council of the Academy of Science, U.S.S.R.

Masses and ages of very distant open clusters for which the colour-magnitude diagram cannot be constructed may be estimated from their integral magnitudes and colours. The network of evolutionary tracks for Population I stars allows a study of evolutionary changes of integral properties of model clusters. Evolutionary tracks for stars with masses $0.8 \mathfrak{M}_{\odot} \leqslant \mathfrak{M} \leqslant 15 \mathfrak{M}_{\odot}(X=0.7, Z=0.03)$ by Paczyński (1970) have been used and isochrones have been derived by an interpolation method. The initial mass function assumed for cluster models is the Salpeter function for 58, 285 and 580 stars. The integral colours and magnitudes were calculated for cluster ages $t=0$ and $6 \leqslant \log t \leqslant 9$ and the ratio $\log \mathfrak{M} /$ integral magnitude has been derived.

Parameters obtained for cluster models with different ages and integral masses have been compared with observational data for clusters with similar characteristics. Correlations $\log \mathfrak{M} /$ integral magnitude for these clusters have been derived on the basis of observations. The agreement of observational data with theoretical results is rather good.

\section{Reference}

Paczyński, B.: 1970, Acta Astron. 20. 47. 